\title{
Studies of heavy-ion collisions with a two-component model.
}

\author{
Alexander BYLINKIN* \\ (Moscow Institute of Physics and Technology (MIPT), Moscow, Russia) \\ E-mail: alexander.bylinkin@gmail.com

\section{Nadezda CHERNYAVSKAYA} \\ (Moscow Institute of Physics and Technology (MIPT), Moscow, Russia) \\ E-mail: nadezda. chernyavskaya@gmail.com
}

\section{Andrey ROSTOVTSEV}

(Institute of the Information Transition Problems (IITP), Moscow, Russia)

E-mail: rostoveitep.ru

\begin{abstract}
Charged particles production in heavy-ions collisions is considered in this paper in terms of a recently introduced Two Component Model. The variations of the parameters of the introduced approach with the center of mass energy and centrality are studied using the available data from RHIC and LHC experiments. The spectra shapes are found to show an universal dependence on the number of participating nucleons $N_{\text {part }}$ for all investigated collision energies. Next, the dependences of the obtained charged hadron densities on the $N_{\text {part }}$ are discussed. The Two Component Model allows to separate the charged hadron densities originating from two distinct mechanisms of hadroproduction. Scaling of "soft" and "hard" contributions to the spectra with centrality is discussed.
\end{abstract}

7th International Conference on Physics and Astrophysics of Quark Gluon Plasma

1-5 February, 2015

Kolkata, India

${ }^{*}$ Speaker. 


\section{Introduction}

Recently a qualitative model considering two sources of hadroproduction [1] and its hydrodynamic extension [2] for the case of heavy-ion collisions have been introduced. For the case of heavy-ion collisions it was suggested to parametrize charged particle spectra by a sum of an exponential (Boltzmann-like) and a power-law $p_{T}$ distributions:

$$
\frac{\mathrm{d}^{2} \sigma}{\mathrm{d} \eta \mathrm{d} p_{T}^{2}}=A_{e} \cdot \int_{0}^{R} r \cdot \mathrm{d} r \cdot m_{T} I_{0}\left(\frac{p_{T} \sinh \rho}{T_{e}}\right) K_{1}\left(\frac{m_{T} \cosh \rho}{T_{e}}\right)+\frac{A}{\left(1+\frac{p_{T}^{2}}{T^{2} \cdot N}\right)^{N}}
$$

where $E_{T k i n}=\sqrt{p_{T}^{2}+M^{2}}-M$ with $M$ equal to the produced hadron mass and $A_{e}, A, T_{e}, T, N$ are the free parameters to be determined by fit to the data. The detailed arguments for this particular choice are given in $[1,2]$. The exponential term in this model is associated with thermalized production of hadrons by valence quarks and a quark-gluon cloud coupled to them. While the power-law term is related to the mini-jet fragmentation of the virtual partons (pomerons in pQCD) exchanged between the colliding partonic systems. In [2] it was also shown that an additional power-law term is needed to describe the charged hadron spectra in central $\mathrm{PbPb}$ collisions. A typical charged particle spectrum as a function of transverse momentum measured by the ALICE collaboration in $\mathrm{PbPb}$ collisions is shown in figure 1 together with the introduced new three component fit function.

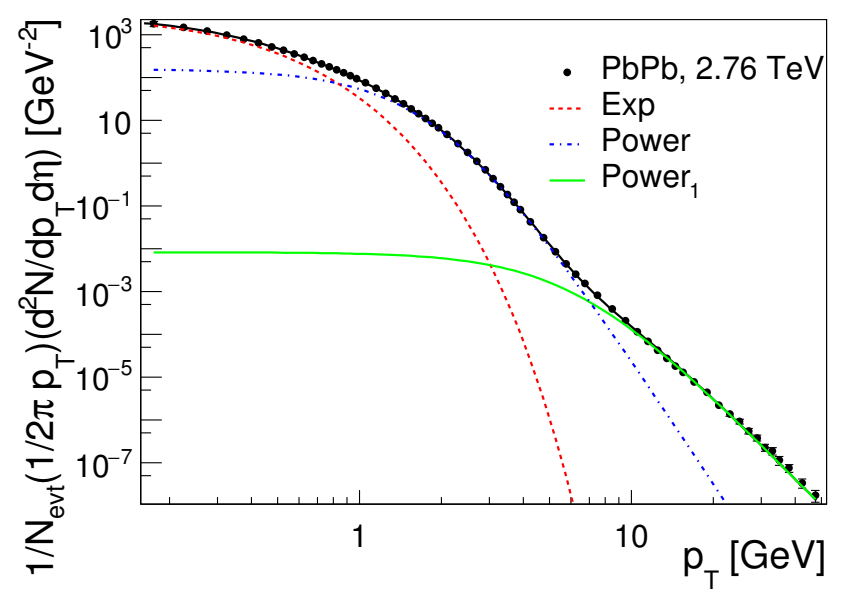

Figure 1: Central lead-lead collisions: the red (dashed) line shows the hydrodynamic term and the green (solid) and blue (dash-dot) lines - two power-law terms.

While the high $-p_{T}$ tail (shown with the green line in figure 1) has the same slope parameter $N$ as in $p p$-collisions and can be rather well reproduced in Monte Carlo generators, the low- $p_{T}$ ("soft" part) and mid- $p_{T}$ ("hard") parts (shown with red and blue lines in figure 1) of the spectra have only phenomenological descriptions. Therefore, in this paper we suggest to look how these two contributions and their parameter values vary with centrality, center of mass energy and pseudorapidity region to better understand the dynamics of charged hadron production in heavy-ion collisions. 


\section{Parameters}

In [2] it was suggested that the charged particle spectra in heavy ion collisions can be divided into several components, corresponding to distinct values of transverse momentum and various mechanisms of hadroproduction. The low- $p_{T}$ region is dominated by the "thermal" particle production (red line in figure 1). For the high- $p_{T}$ tail the constant suppression $\left(R_{A A}\right.$ value) is observed, indicating that the slope parameter " $\mathrm{N}$ " of the power-law term (green line in figure 1) should obtain the same value for any centralities as in $p p$-collisions at the same c.m.s. energy and should stand for the point-like parton-parton collisions. In this section we then suggest to have a closer look at the mid- $p_{T}$ contribution (blue line in figure 1) and the variations of the $T$ and $N$ parameters, which are expected to differ both with the collision energy and centrality.

The mini-jets travelling through the dense medium should loose their energy because of the multiple rescatterings. This effect, in turn, will result in higher values of $T$ and $N$ parameters (of the mid- $p_{T}$ term), as expected for higher thermalization. Here we consider $p p$-collisions as a reference point for the case of heavy-ion collisions to study the modification of the spectra with centrality. On the other hand, the most universal parameter characterizing the centrality of a heavy-ion collision is $N_{\text {part }}$ or the number of participating nucleons related to the impact parameter. Therefore, we propose to look at the variation of the ratios $T / T_{p p}$ and $N / N_{p p}$ obtained from the fit to the experimental data as a function of $N_{p a r t}$, where $T_{p p}$ and $N_{p p}$ stand for the parameter values obtained for $p p$-collisions at the same c.m.s. energy per nucleon $\sqrt{s} / N$.

As shown in figures 2, indeed, both of the parameters, $T$ and $N$, grow with $N_{\text {part }}$ as it was natural from our naive expectations. Using the data from the ALICE [3], ATLAS [4] and PHENIX [5] experiments the behaviour of the parameters can be parametrized in the following way as a function of $N_{\text {part }}$ :

$$
N / N_{p p}=0.02 \cdot N_{\text {part }}^{0.53}+0.97, \quad T / T_{p p}=0.07 \cdot N_{\text {part }}^{0.30}+0.91 .
$$

Remarkably, the same universal scaling of these parameters with $N_{\text {part }}$ is observed for both RHIC and LHC data.
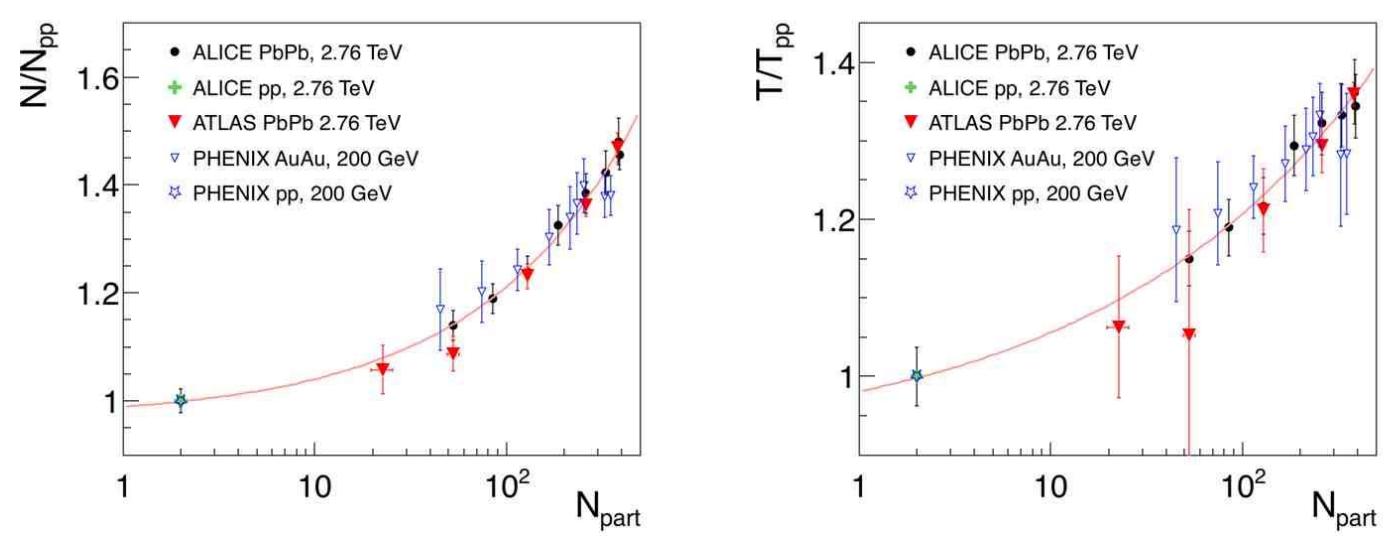

Figure 2: The dependencse of the ratios of the parameters $N$ over $N_{p p}$ and $T$ over $T_{p p}$ on $N_{p a r t}$. The $T_{p p}$ and $N_{p p}$ are determined for pp-collisions at the same c.m.s. energy. 


\section{Charged hadron densities}

Let us now have a look how charged hadron densities $d N_{c h} / d \eta$ vary with the centrality of heavy-ions collisions. They are expected to scale with $N_{\text {part }}$ or $N_{\text {coll }}$ for "soft" and "hard" regimes of particle production, respectively, and this scaling becomes a subject of vairous phenomenological discussions: linear scaling with $N_{\text {part }}$ is ecpected for "soft" processes, while scaling with $N_{\text {coll }}$ is expected for the "hard" regime of particle production [6] ${ }^{1}$. Therefore, to study this scaling we suggest to plot charged hadron densities $1 / N_{\text {evt }} d N / d \eta / N_{\text {part }}$ separately for "soft" and "hard" contributions of our model to the spectra as functions of $N_{\text {part }}$ as shown in the figure 3 .
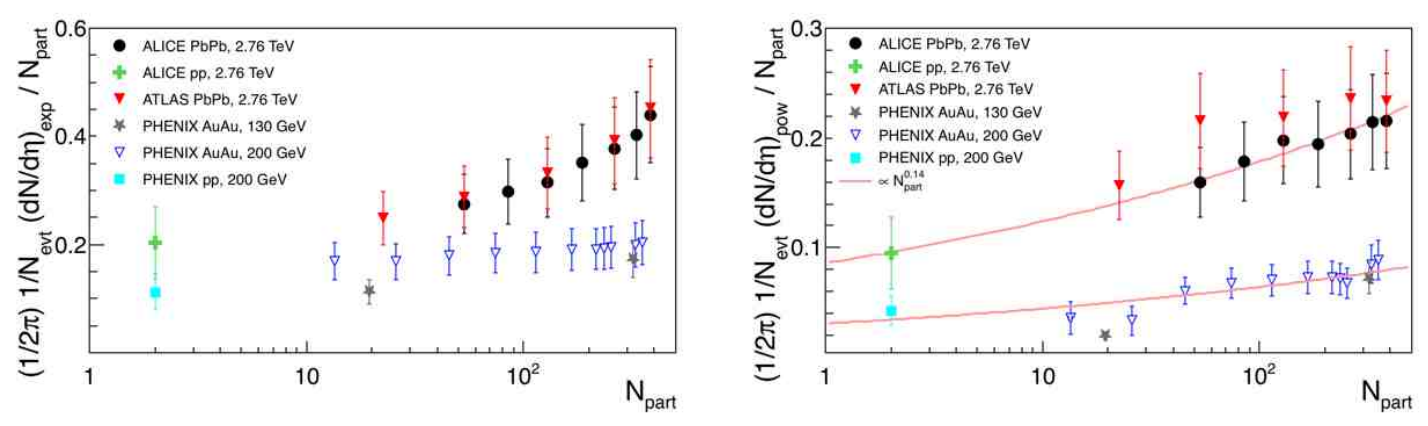

Figure 3: The charged particle densities divided over the number of participating nucleons $N_{\text {part }}$ obtained for "thermal" and "hard" parts of the spectra as functions of $N_{\text {part }}$.

As one can notice in figure $3 \mathrm{~b}$ ) charged hadron densities obtained for the power-law term contribution show an universal scaling with $N_{\text {part }}^{\alpha}$ independent on collision energy, with $\alpha>1$ indicating that indeed the power-law term of our model can be related to some "hard" regime of hadron production. On the other hand, in figure 3 a) the linear scaling with $N_{\text {part }}$ (that can be expected for the exponential term of our model) is observed only for RHIC and not for LHC data, with the enhancement at large $N_{\text {part }}$ values seen for the latter.

Such behavior might be explained by the following hypothesis using the idea of QGP to hadron-gas phase transition and its critical temperature $T_{c}$. At low collision energies or in peripheral heavy-ion interactions the dense matter formed has the temperature below the critical value, thus the number of final-state hadrons is proportional to the number of partons involved in the interaction. However, for central lead-lead collisions at LHC the quark-gluon matter reaches the the temperatures above the critical value. Since the hadrons can be produced with a temperature not higher than $T_{c}$, the phase transition takes place and taking into account the energy conservation law one should observe the enhancement of the number of produced particles in this case.

Let us now check this hypothesis. First of all, in 4 a) the variations of the temperature, $T_{e}$, of the "thermal" hadron production are shown: indeed collisions at RHIC result in the temperature close to the critical one only for the most central gold-gold interactions, while for LHC the freeze-out temperature is observed. This fact is already in accordance with the qualitative picture described above. To perform the quantitative test we suggest to calculate the total transverse energy $d E_{T} / d \eta$ for "thermal" part of the spectra. This quantity divided over the number of participants is shown in

\footnotetext{
${ }^{1}$ Also note that $N_{\text {coll }} \propto N_{\text {part }}^{4 / 3}$.
} 
figure $4 \mathrm{~b}$ ). One can see that the total transverse energy $d E_{T} / d \eta$ has the same scaling with $N_{\text {part }}$ for both RHIC and LHC data which confirms the hypothesis made.
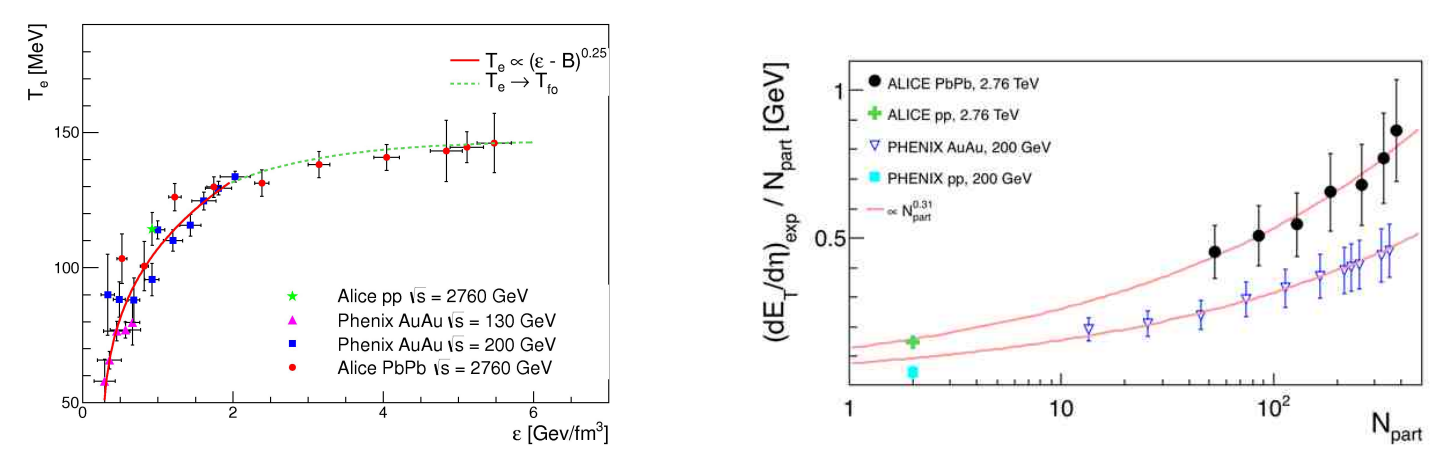

Figure 4: The temparature variations $T_{e}$ as a function of energy density (a) and the total transverse energy for "soft" particle production over the number of participating nucleons $N_{\text {part }}$ as a function of $N_{\text {part }}$ (b).

Finally, one can calculate the nuclear modification factor $R_{A A}$ for any centralities and energies using the dependences observed together with the results obtained in [7]. Such predictions together with two sets of ALICE data are shown in figure 5 both for central and peripheral collisions.

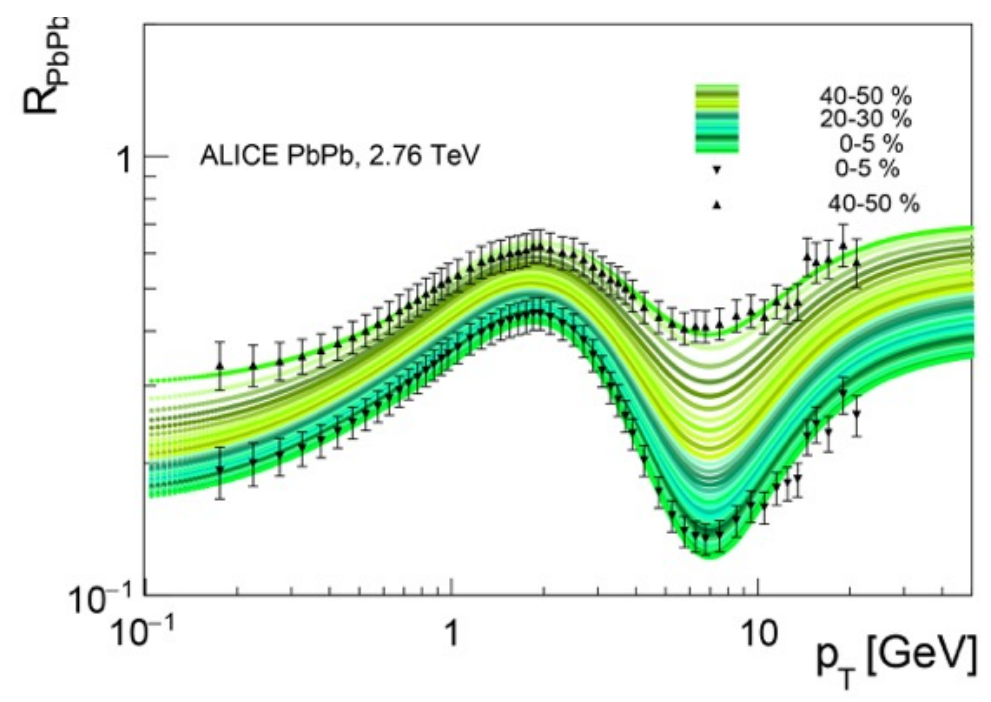

Figure 5: Prediction on $R_{A A}$ together with two sets of ALICE data.

\section{Conclusion}

In conclusion, transverse momentum spectra in heavy ion collisions have been considered using the Two Component Model. The variations of the parameters obtained from the fit have been studied as a function of $N_{\text {part }}$ and c.m.s. energy. The same universal dependence of the parameters of the model $T$ and $N$ for RHIC and LHC data was found. Next, scaling of "soft" and "hard" contribution to the charged hadron spectra with centrality in heavy-ion collisions were studied 
separately. The charged hadron densities for "hard" part turned out to have the same scaling for RHIC and LHC data with $\alpha>1$. For the "soft" part the deviation from $\alpha \approx 1$ was found for LHC and explained by the phase transition taking place in these collisions. Finally, the predictions on the shape of $R_{A A}$ have been made.

\section{References}

[1] A. A. Bylinkin and A. A. Rostovtsev, Phys. Atom. Nucl. 75 (2012) 999 Yad. Fiz. 75 (2012) 1060; A. A. Bylinkin and A. A. Rostovtsev, arXiv:1008.0332 [hep-ph].

[2] A. A. Bylinkin, N. S. Chernyavskaya and A. A. Rostovtsev, Phys. Rev. C 90 (2014) 1, 018201 [arXiv:1405.3055 [hep-ph]].

[3] B. Abelev et al. [ALICE Collaboration], Phys. Lett. B 720 (2013) 52 [arXiv:1208.2711 [hep-ex]].

[4] G. Aad et al. [ATLAS Collaboration], arXiv:1504.04337 [hep-ex].

[5] S. S. Adler et al. [PHENIX Collaboration], Phys. Rev. C 69 (2004) 034910 [nucl-ex/0308006].

[6] B. Abelev et al. [ALICE Collaboration], Phys. Rev. C 88 (2013) 4, 044909 [arXiv:1301.4361 [nucl-ex]].

[7] A. Bylinkin, N. S. Chernyavskaya and A. A. Rostovtsev, Eur. Phys. J. C 75 (2015) 4, 166 [arXiv:1501.05235 [hep-ph]]. 